\title{
Atypical Ductal Hyperplasia of the Breast: Management of a Clinical Case at the IBN SINA Hospital Centre with Literature Review
}

\author{
Jean Alfred M'bongo*, Yacir El Alami, Wilfried Loïc Tatsipie Meukem, Saissi Ananas, \\ Fouad Tijami, Hanchi El Zaki, Hachi Hafid
}

Gynecomammary Surgery Department, Rabat, Morocco

Email: *mbongojalf@gmail.com

How to cite this paper: M’bongo, J.A., El Alami, Y., Meukem, W.L.T., Ananas, S., Tijami, F., El Zaki, H. and Hafid, H. (2019) Atypical Ductal Hyperplasia of the Breast: Management of a Clinical Case at the IBN SINA Hospital Centre with Literature Review. Open Journal of Obstetrics and $G y$ necology, 9, 1161-1167.

https://doi.org/10.4236/ojog.2019.98112

Received: May 10, 2019

Accepted: August 16, 2019

Published: August 19, 2019

Copyright $\odot 2019$ by author(s) and Scientific Research Publishing Inc. This work is licensed under the Creative Commons Attribution International License (CC BY 4.0).

http://creativecommons.org/licenses/by/4.0/

\begin{abstract}
Objective: To report a case of difficulties in the management of atypical ductal hyperplasia (ADH). Presentation of the case: Mrs. G, 50 years old, is consulting following the discovery at autopalpation of a lesion on her left breast. In its history: radical mastectomy Right Patey in 2004 for ductal carcinoma Infiltrant associated with carcinoma in situ; $2 \mathrm{~N}+$ /14; Positive hormone receptors. Adjuvant treatment performed: chemotherapy, radiotherapy and hormone therapy. Summary of the clinical case: Left breast examination: Superior External Quadrant nodule $5 \mathrm{~cm} \times 4$, mobile, hard, without inflammatory signs, there is no palpable lymph node. The surgical scar of the right breast is without particularity. Mammography and left breast ultrasound show an ACR4 lesion according to BIRADS. Microbiopsy: intradural papillomatous lesion requiring verification of the myoepithelial layer (P63 and CK5/6). Immunohistochemistry: atypical ductal hyperplasia (ADH) with no sign of transformation. Normal CA15-3 dosage. Treatment: broad surgical removal of the lesion. Analysis of the part shows a lesion with all the criteria of an HCA measuring $2 \mathrm{~mm}$ in its largest axis. The postoperative consequences are simple. Conclusion: The management of atypical hyperplasia is not consensual and is often undervalued. The type of lesion characterizing HCA is decisive for therapeutic orientation.
\end{abstract}

\section{Keywords}

Atypical Ductal Hyperplasia, Management

\section{Introduction}

Atypical ductal hyperplasia (ADH) is an intra-channel monomorphic cell proliferation with certain cytological and architectural characteristics of ductal carcinomas in situ (CCIS) [1]. The variable clinical significance between marker 
and precursor of breast cancer, of these atypical hyperplasias is discussed; they are classified into three types: HCA, lobular neoplasia (NL), and cylindrical metaplasia with atypia [1]. The discovery of HCA is often fortuitous on breast tissue [1], and management is not consensual [2]. There are no specific recommendations on the technique for removing atypical hyperplasia lesions discovered on percutaneous biopsy; these lesions are often subclinical. This surgery requires preoperative identification [1]. Many advances have been made in their histological diagnosis, classification and pathogenesis, but there is still no consensus on their management, particularly surgical management, which remains controversial [3]. We report one case with a literature review.

\section{Clinical Case}

Mrs. G, 50 years old, single, nulligest, nulliparous, consults January 26, 2019 for left breast ball at autopalpation. She has no notion of oral contraception or cancer in the family, but a history of right Patey mastectomy in 2004. Histology confirmed a multifocal invasive ductal invasive carcinoma with carcinoma in situ, the surgical limits were healthy. Axillary cleaning had reported $2 \mathrm{~N}+/ 14$ (2 positive nodules out of 14). The hormone receptors were positive. The treatment received was limited to chemotherapy, then radiotherapy and hormone therapy for five years. No secondary complications were noted during this period and during the five years following the discontinuation of her treatment during the surveillance, and she was then lost sight of until the day of her consultation.

Upon admission, the general condition is preserved. But the patient is anxious because of the cancer.

On physical examination, the patient presented at the left contralateral breast level, a mass of the superior external quadrant of $3 \mathrm{~cm}$ in diameter, mobile, hard, without inflammatory signs. The surgical scar of the contralateral breast is without particularity, the rest of the clinical examination shows no particularity. At para-clinical exploration, the CA15-3 assay was normal. Mammography (Figure 1 and Figure 2) and left breast ultrasound show a BIRADS 4 lesion, while micro-biopsy shows an intradural papillomatous lesion requiring verification of the myoepithelial layer (P63 and CK5/6). Immuno-histochemistry, is in favor of atypical ductal hyperplasia (HCA) by confirming myoepithelial and epithelial cells that are without immunolabelled atypia at CK 5/6.

Therapeutic management, with the patient's informed consent, required a multidisciplinary consultation meeting or a surgical decision was made.

The treatment was broad surgical excision with oncoplasty by the external technique which made it possible to remove the tumor and skin facing away from the body, to readapt the skin sheath to the new glandular volume and thanks to the repositioning of the areola at the top and inside to avoid it being attracted outside.

The postoperative outcomes were simple, anxiety disappeared and oncoplastic surgery with a flap of the large dorsal is planned for the right breast to improve the aesthetic appearance of the breasts and the patient's femininity. 


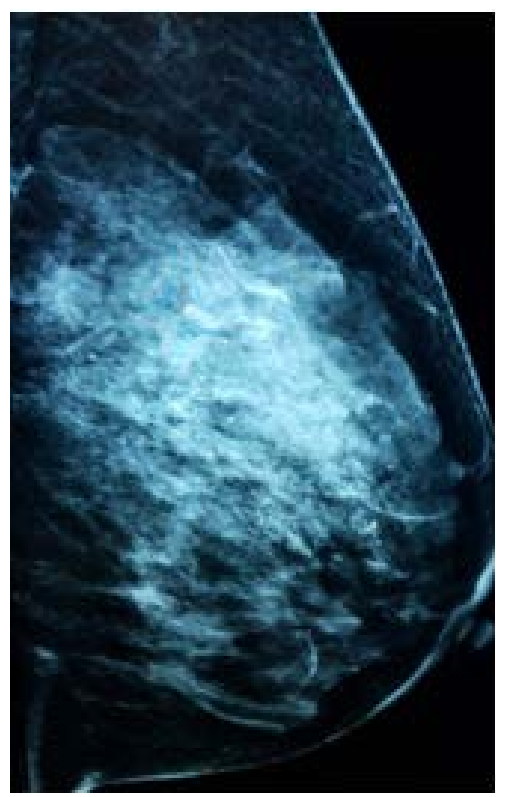

Figure 1. Micro-calibrations (Front view).

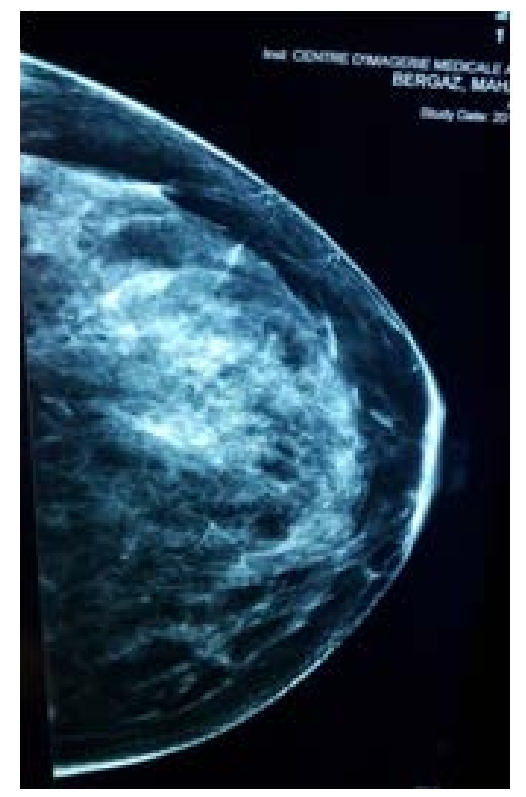

Figure 2. Micro-calibrations (Front view).

A complete analysis of the operating room shows a lesion with all the criteria of an HCA measuring only $2 \mathrm{~mm}$ of the largest axis.

\section{Discussion}

In our clinical case, the diagnostic referral to $\mathrm{ADH}$ was the presence of micro calcifications on mammography that corroborate some assertions in the literature [1] [4]. However, HCAs do not have a specific radiological translation, the most frequent sign of call is that of an isolated micro calcification site, histological evidence is obtained by macro biopsy to target the micro calcification site [5]. 
Histological diagnosis is difficult, with great inter-observer variability. Breast samples obtained by the per cutaneous route are small and fragmented, leading to underestimation of lesions and variability in false-negative rates depending on the techniques used (needle size, aspiration system) [5] [6]. For some authors, histological evidence is obtained by stereotactic macrobiopsy, making it possible to target micro calcification foci by calculating spatial coordinates on so-called stereotactic clichés [5]. Sometimes, atypical hyperplasia result in abnormal masses or ranges on ultrasound, in magnetic resonance imaging (MRI), an increase in focal distribution mass can be observed after injection of gadolinium [5]. The "atypical" nature of hyperplasia has been established by immunohistochemistry as reported by some authors [7].

In front of an $\mathrm{ADH}$, doubt is a source of fear: lifting doubt by monitoring or operating to have the lesion as a whole? In $\mathrm{ADH}$, the carcinological risk corresponds to the probability of malignant degeneration of the lesion [8]. There are several of them:

Histological risk (increase in relative risk in case of $\mathrm{ADH}$ ), genetic risk (BRCA1 and BRCA2 mutation), age-related risk (breast cancer incidence increases sharply from 35 to 50 years with a peak at 60 years), radiological risk (the image gives a diagnostic presumption, the evidence being provided by histology) [8]. For some authors [3], the risk of breast cancer occurring later is 4 to 5 times higher than in the control population, as this cancer can affect both the same breast and the lateral control breast. HCA is often associated with pejorative lesions of the ductal carcinoma in situ and invasive carcinoma type, for this reason, some authors consider ADH as a precursor to invasion [9], it would seem that epithelial atypia is a marker of both concomitant and secondary cancer risk [10]. However, compared to the general population, the relative risk of breast cancer after $\mathrm{ADH}$ is less than 5, whether on surgical biopsy or with a needle [11]. Other authors question whether there are arguments to assert pre-cancerous lesions, if they are not, surgical removal of the breast will have no preventive role [12].

Due to the personal history of breast cancer, the patient's age, the localized nature of the lesion, and psychological factors (the patient's cancer phobia), we performed extensive excision. Indeed, surgery allows the analysis of the entire part to eliminate false negatives [1] [7]. For some authors, when the lesion is localized, excision surgery is the most appropriate therapeutic method; however, when they are extensive or bilateral, management is more difficult [8]. In the case of high-risk lesions requiring a surgical decision, when skin samples are diagnosed, a diagnostic zonectomy is performed for a complete morphological assessment of the operating part in order to look for a possible lesional multifocality, quantification of the neoplastic charge or association of carcinoma territories in situ or infiltrating [13]. For others, the indication for prophylactic mastectomy is highly controversial, based exclusively on the assessment of a "high risk" [1].

The recommendations of the Léon Bérard Center (CLB) [14] 

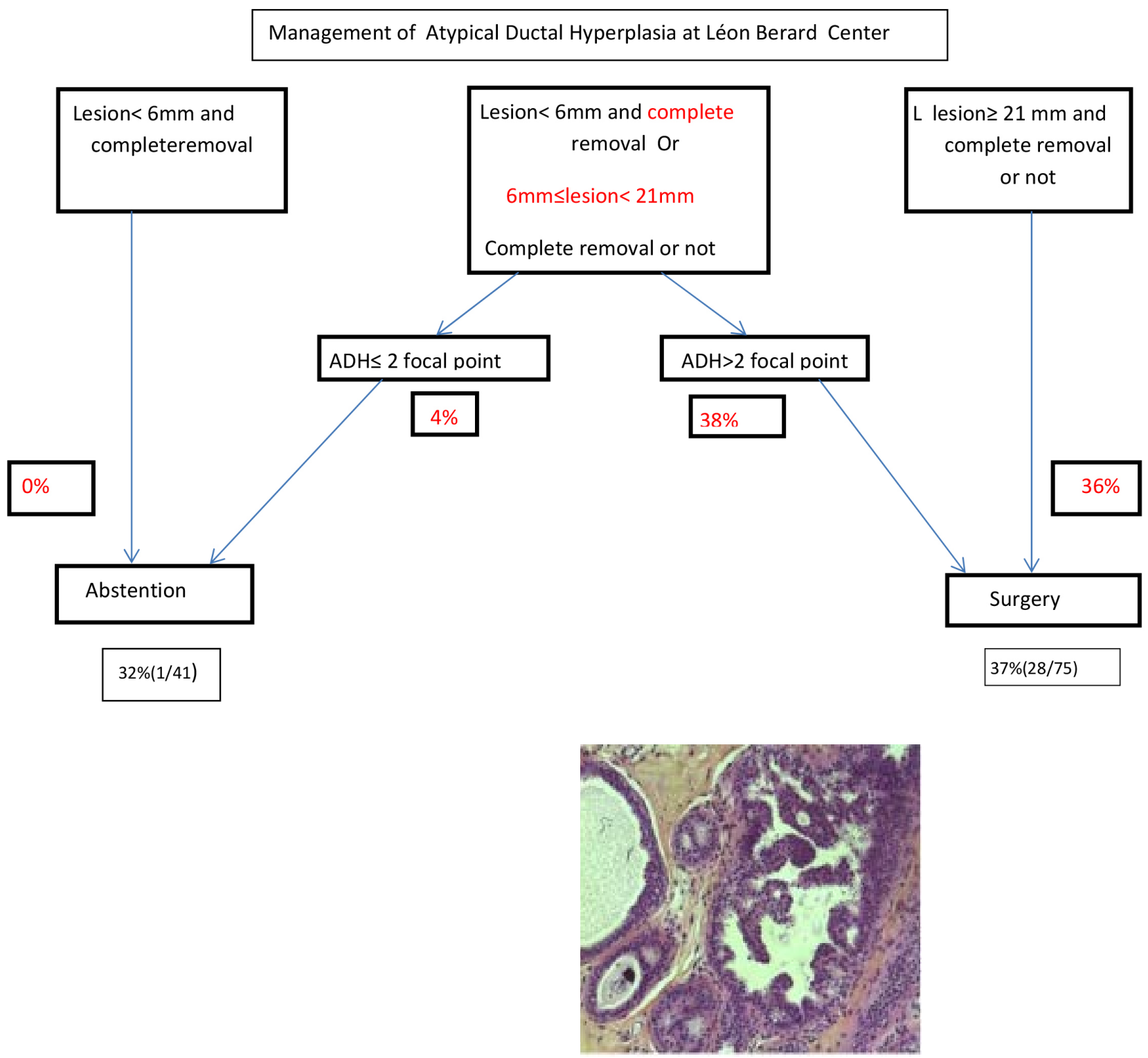

Figure 3. Proliferation of monomorphic cells within a pathological channel Immunolabeling with ck5/6.

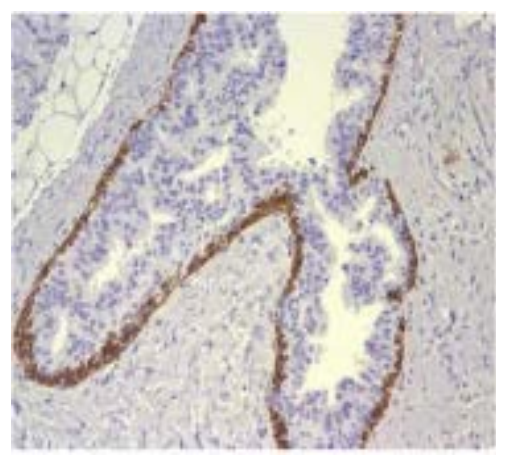

Figure 4. Only myoepithelial and epithelial cells without atypia. 
If an atypical lesion is found on a biopsy, the current standard attitude is to perform an exeresis [2]. In the postoperative period, we did not recommend any particular treatment. Some authors use hormone therapy. Hormone therapy has been evaluated, the benefit-risk balance does not encourage its prescription [15], in fact, hormone replacement therapy in a patient with atypical hyperplasia increases the relative risk of breast cancer [16] (Figure 3, Figure 4).

In case of surveillance, the clinical examination is carried out every 6 months for 2 years, then every year; the annual unilateral or bilateral mammography and possibly the ultrasound after a period of 10 years [17].

\section{Conclusions}

The management of atypical hyperplasia is not consensual and is often undervalued.

The type of lesion characterizing $\mathrm{ADH}$ is decisive for therapeutic orientation towards either surveillance or prophylactic mastectomy.

\section{Conflicts of Interest}

The authors declare no conflicts of interest regarding the publication of this paper.

\section{References}

[1] Washed, V., Bertel, C., Tas, P., Rouquette, S., D’Hallirin, F., Blanchot, J. and Leveque, J. (2008) Surgery for Atypical Hyperplasia. CNGOF, Paris, 32nd National Days.

[2] Washed, V., Bertel, C., Tas, P., Bendavid, C., Rouquette, S., Foucher, F., Audrain, O., Bouriel, C. and Leveque, J. (2010) Atypical Epithelial Hyperplasia of the Breast: Knowledge Assessment and Clinical Practice. Journal de Gynécologie Obstétrique et Biologie de la Reproduction, 39, 11-24. https://doi.org/10.1016/j.jgyn.2009.09.007

[3] Beverage-Lacroix, M., Huntervent-Lobrot, G., Ferrin, S., Lippa, N. and Mac Grognan, G. (2015) “At Risk" Lesions and Borders High-Risk Lesion. Female Imaging, 25, 88-97. https://doi.org/10.1016/j.femme.2015.03.008

[4] Travadé, A., Isnard, A., Bouchet, F. and Bagard, C. (2006) Impalpable Breast Lesions and Stereotactic Macrobiopsy with Mammotome 11G: Should It Be Operated after Diagnosis of Atypical Ductal Hyperplasia? Journal of Radiology, 87, 307-310. https://doi.org/10.1016/S0221-0363(06)74005-7

[5] Journo, G. (2017) Management of Atypical Ductal Hyperplasia: Relevance of the Decision Tree of the CLCC of Lyon, Retrospective Analysis on a Series of Patients from the Institute Curie. Human Medicine and Pathology. https://dumas.ccsd.cnrs.fr/dumas-01737640

[6] Balu-Maestro, F., Eltore, C., Chapellier, I., Peyrottes, P. and Le Blanc, T. (2006) When to Be Wary of the Results of Micro and Macro Guided Mammary Biopsies? Journal of Radiology, 87, 265-273. https://doi.org/10.1016/S0221-0363(06)74000-8

[7] Delaloye, J.F. and Leher, H.A. (2006) Should All Pre-Malignant Breast Lesions Be Operated on? Revue Médicale Suisse, 2, 317-318.

[8] Lazrak, I., Babahabib, A., Kouach, J., Moussaoui, D. and Dehayui, M. (2014) Breast Lesions Called "Boder-Line": Diagnostic and Therapeutic Management. International Journal of Innovation and Applied Studies, 7, 736-747. 
[9] Collins, L.C., Achacoso, N.A., Neklyndov, L., Fletcher, S.W., Haque, R., Quessenberry, C.P., et al. (2007) Clinical and Pathologic Features of Ductal Carcinoma in Situ Associated with the Presence of Flat Epithelial Atypia: An Analysis of $543 \mathrm{~Pa}$ tients. Modern Pathology, 20, 1149-1155.

https://doi.org/10.1038/modpathol.3800949

[10] Sabelle, P., Mascarel, G. and Grogan, M. (2007) Management of Epithelial Atypia of the Breast. Annals of Pathology, 27, 182-194.

[11] Drouet, Y., Le Gall, J., Gervasoni, J., Peix, M., Faure, C., Lasset, C. and Treuilleux, I. (2018) Management of Atypical Ductal Hyperplasia on Macrobiopsy: A Possible Climb? Imaging of the Woman, 28, 138-143. https://doi.org/10.1016/j.femme.2018.03.005

[12] Antoine, M., Teilhac, M.F., Poulet, B. and Cros, J. (2009) Atypical Hyperplasia: Risk Factors or Precancerous Lesion? CNGOF, Paris, 33rd National Days.

[13] Croce, S., Pretz-Grenier, M.F. and Mathelon, C. (2008) The Main Benign and At-Risk Epithelial Breast Lesions. Diagnostic and Therapeutic Management. Gynecology Obstetrics \& Fertility, 36, 788-799. https://doi.org/10.1016/j.gyobfe.2008.02.029

[14] Tardivon, A. and Faure, C. (2015) Lesions at Risk: Does CPR Have Its Place? 37 th Day of the French Society of Senology and Breast Pathology, Bordeaux, 11-13 November 2015.

[15] Fisher, B., Constantino, J.P., Wickerham, D.L., Redmond, C.K., Kavanah, M., Cronon, W.M., et al. (1998) Tamoxifen for Prevention of Breast Cancer: Report of the National Surgical Adjuvant Breast and Bowel Project P-1 Study. Journal of the National Cancer Institute, 90, 1371-1388. https://doi.org/10.1093/jnci/90.18.1371

[16] Fournier, A., Benino, F. and Clavel-Chapelon, F. (2008) Unequal Risks for Breast Cancer Associated with Different Hormone Replacement Therapies: Results from the E3N Cohort Study. Breast Cancer Research and Treatment, 107, 103-111. https://doi.org/10.1007/s10549-007-9604-x

[17] Scemama, O. and Dulaveyne, R. (2018) Breast Cancer: Specific Screening Modalities for High-Risk Women. HAS News and Practices. http://www.has-santé.fr 